\title{
Robust design of valve spring based on Taught method En'guo Dong ${ }^{1, a}$, Lei Zhang ${ }^{1, b}$
}

${ }^{1}$ Automotive and Transport School, Tianjin University of Technology and Education, Tianjin, China, 300222

adegzl@126.com, bzldeg@sohu.com

Key words: Valve spring; robust design; Taught Method; Monte Carlo method

\begin{abstract}
Existing valve spring designs are using the deterministic optimization method without considering the uncertainties in manufacturing and assembling process. We propose to take them into account at an early design stage in order to ensure robustness which is less sensitive to the uncertainties of design parameters. A mathematic model on a valve spring is performed. The optimization objective is minimum mass and higher defending resonate performance. Optimization results are solved using the Genetic Algorithm. Considering the random error, the robustness of the optimization results on the valve spring is analyzed and the valve spring is not robust. The valve spring is designed again using the Taguchi method and the robustness is improved.
\end{abstract}

\section{Introduction}

A valve spring is a cylindrical helical spring, which is in general designed by a traditional optimization method. However, design results based on the traditional optimization method are often at the edge of the value range, and a tiny uncertainty change may lead to design failure. Therefore a traditional deterministic optimization method is not enough to ensure the robustness of result. In the early design process, the Monte Carlo method can be used to rectified the product quality, and reduces the product loss as in [1-2]. Therefore, considering the uncertainties of design variables, the robustness of a valve spring is analyzed by the Monte Carlo method. Moreover, the valve spring is designed again using the Taguchi method to improve robust performance.

\section{Mathematical model on valve spring}

\section{Objective function}

The design goal is the minimum mass and the best defending resonance of valve spring as in [3] and is expressed

$$
F(x)=\omega F_{1}(x)+(1-\omega) F_{2}(x)
$$

where, $F_{1}(x)=\frac{\left(n+n_{2}\right) \pi D d^{2} \rho}{4}$ is the spring mass. $\rho$ is the steel wire density, $n_{2}$ is the support turns of spring, $d$ is the steel wire diameter, $D$ is the spring diameter, and $n$ is the spring working turns; $F_{2}(x)=\frac{1}{f}=\frac{n D^{2}}{3.56 \times 10^{5} d}$ is the spring frequency. $\omega$ is the weight coefficient, and the value is 0.2 .

\section{Constrain conditions}

The constraints are expressed in a set of equations as followings. Equation (2) is the spring index; equation (3) is the strength condition; equation (4) is the stability condition; equation (5) is the minimum working turns; equation (6) is the spring diameter range; equation (7) is the steel wire diameter range.

$$
\begin{aligned}
& 4 \leq \frac{D}{d} \leq 14 \\
& \frac{8 K P D}{\pi d^{3}}-[\tau] \leq 0
\end{aligned}
$$


$\frac{\left(n+n_{2}-0.5\right) d+1.1 \lambda}{D}-5.3 \leq 0$

$3-n \leq 0$

$30 \leq D \leq 40$

$2.5 \leq d \leq 9.5$

Where, $P$ is the load; $K$ is the curvature coefficients.

Design results on valve spring

The load of valve spring is $680 \mathrm{~N}$, and the maximum deformation is $16.59 \mathrm{~mm}$, the working frequency is $25 \mathrm{~Hz}$, and the material is $50 \mathrm{GrVA}$.

Three parameters of the wire diameter $(d)$, the spring diameter $(D)$ and the spring turns $(n)$ are defined as design variables and optimization results are solved using the genetic algorithm. The final results are: $[d, D, n]=[7.226,30.128,3.008]$. The constraint conditions and the optimization value are shown as in table 1.

Table 1 Design results on valve spring

\begin{tabular}{|c|c|c|c|c|}
\hline Mass[g] & Resonance[1/Hz] & Spring index & Strength[Mpa] & Stability \\
\hline 47.33 & 0.0011 & 4.1695 & 191.4 & 1.6988 \\
\hline
\end{tabular}

\section{The valve spring robustness analysis based on the Monte Carlo method}

\section{Monte Carlo method}

The basic steps based on the Monte Carlo method as in [4-5] are as the followings:

(1)Determine the response surface model, expressed as the hyper surface $f(x)=f\left(x_{1}, x_{2}, \cdots, x_{n}\right)$ of variable $X$;

(2)Obtain the sample $x^{(j)}=\left\{x_{j 1}, x_{j 2}, \cdots, x_{j n}\right\}^{T}$ by carrying out the random sampling;

(3)Choose the distributed on each independently random variable, including mean and standard deviation of each independently random variable, etc.;

(4)Substitute the sample $x^{(j)}$ into the relevant response surface to get error samples. That constitutes an experiment $\Delta_{\max }^{i}=g\left(x^{j}, \theta_{i}\right)=g\left\{x_{j 1}, x_{j 2}, \cdots, x_{j n}, \beta_{i}\right\}^{T},\left(\beta_{i}\right.$ is the response surface values, $i=1$, $2, \ldots, n)$;

(5)Examine whether or not to meet function $Y_{j}=\Delta_{\max }^{i}-[\Delta] \leq 0 .[\Delta]$ is the maximum permissible error;

(6)Repeat $K$ times from step (2) to step (5), and calculate the numbers of $Y_{j} \leq 0$ in the independently sampling trials based on equation $K_{T}=\sum_{j=1}^{K} U_{j}$.where, $K_{T}$ is the numbers of $Y_{j} \leq 0, U_{j}=\left\{\begin{array}{l}0, Y_{j}>0 \\ 1, Y_{j} \leq 0\end{array}\right.$

(7)Calculate the reliability using function $P(K)=P\left\{\Delta_{\max }-[\Delta] \leq 0\right\}=R_{K} / R$ after the experiment of $K$ times. If the reliability is meet $P(K) \geq \delta$ ( $\delta$ is the allowing reliability), the product quality is robust, otherwise to modify the random variable precision.

(8)Draw probability distribution chart.

\section{The valve spring robustness analysis based on the Monte Carlo method}

Build response surface models on objective function of the spring quality (Weigh), the spring vibration frequency (Resonate). The variables of steel wire diameter $(d)$, spring diameter $(D)$ and spring turns $(n)$ are considered as independent random variables with the normal distribution, and the distributions are: $d \sim N\left(7.226,0.01^{2}\right), \quad D \sim N\left(30.128,0.01^{2}\right), n \sim N\left(3.008,0.03^{2}\right)$.

Robustness analysis based on the Monte Carlo method is carried out. After the random trial of 700 times, the probability density distribution on the response surface models are gotten. The distributions are shown as in Fig.1 and respective are: Weigh $\sim N\left(47.30,1.36^{2}\right)$, Resonate $N\left(0.0011,0.0000396^{2}\right)$. The maximum variation of the spring mass is $2.87 \%$, with the spring 
vibration frequency is $3.6 \%$.
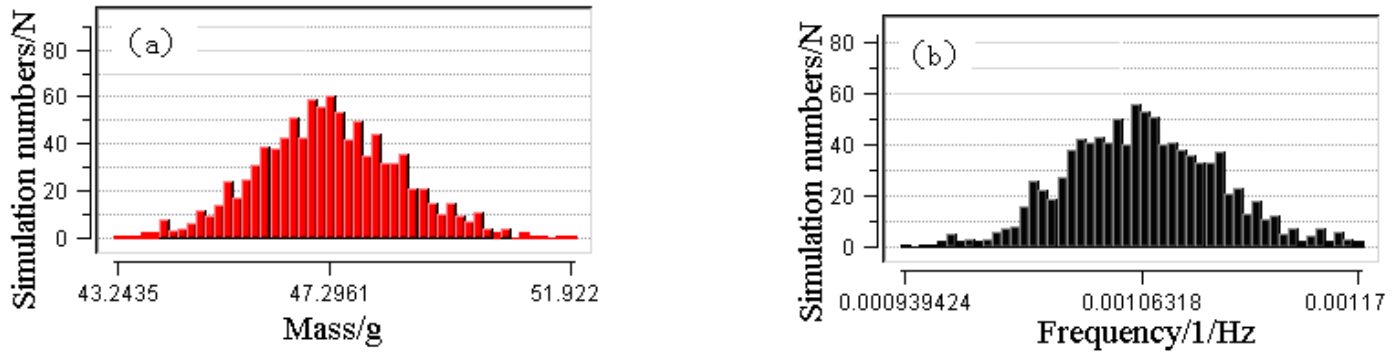

Fig.1 Probability density distribution

(a) Spring mass; (b)Spring frequency

In the design, the vibration frequency does not meet the design requirement of less $3 \%$. Therefore the valve spring need be designed again.

Figure 2 is the key factor analysis. As can be seen, the key factors on the spring mass are the steel wire diameter, the spring diameter, the spring turns in order. The key factors on the vibration frequency are the spring diameter, the steel wire diameter, and spring turns. Based on the analysis, the robust design of the spring is carried out. The controlled factors are the steel wire diameter and the spring diameter, and the noise factors are the spring turns.
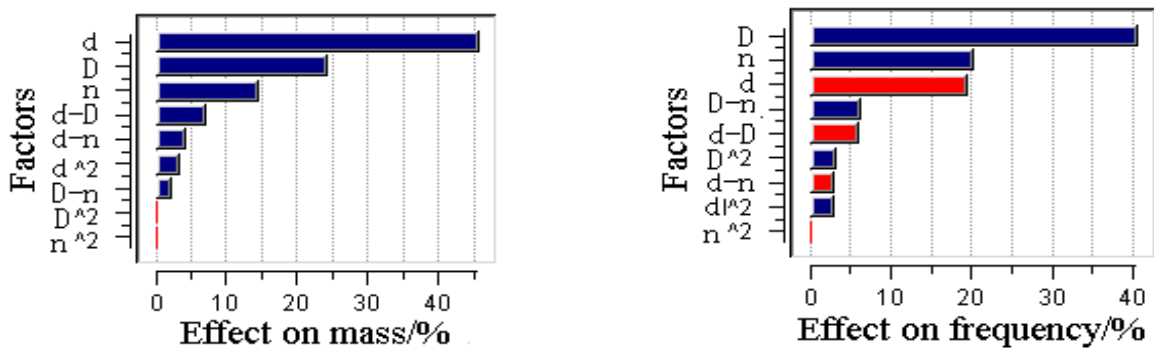

Fig.2 Key factor analysis

\section{The robust design based on Taguchi method}

\section{Design model}

After the test of 27 times, the results are obtained as $[d, D]=[7.1537,29.8267]$. The mean of the mass is $45.87 \mathrm{~g}$, with the variance of $0.0011 \mathrm{~g}^{2}$. And the mean of the frequency is $1.366 \mathrm{~Hz}$, with the variance of nearly $0 \mathrm{~Hz}^{2}$.

The controlled factors of the wire diameter, the spring diameter are noted $X=\left[x_{1}, x_{2}\right] \mathrm{T}=[d, D]^{\mathrm{T}}$; and the noise factor of the spring turns is noted $Z=z_{1}=n$. The level table of the controlled factors is shown as in table 2 .

In the orthogonal experiment design, a internal form is as the table of $\mathrm{L}_{9}\left(3^{2}\right)$ and external form is as the table of $\mathrm{L}_{3}\left(3^{1}\right)$.

After a test of 27 times, results are obtained as $[d, D]=[7.1537,29.8267]$. The mean of mass is $45.87 \mathrm{~g}$, with the variance of $0.0011 \mathrm{~g}^{2}$. And the mean of frequency is $1.366 \mathrm{~Hz}$, with the variance of nearly $0 \mathrm{~Hz}^{2}$.

\section{Robust analysis}

The robust analysis is performed again based on the Monte Carlo method. The variable distributions are $: d \sim N\left(7.1537,0.01^{2}\right), D \sim N\left(29.8267,0.01^{2}\right)$.

Tab.2 Controlled factors

\begin{tabular}{|c|c|c|}
\hline \multirow{2}{*}{ Level } & \multicolumn{2}{|c|}{ Factors } \\
\cline { 2 - 3 } & $d[\mathrm{~mm}]$ & $D[\mathrm{~mm}]$ \\
\hline 1 & 7.15374 & 29.8267 \\
\hline 2 & 7.226 & 30.128 \\
\hline 3 & 7.29826 & 30.4293 \\
\hline
\end{tabular}

Tab.3 Uncontrolled factor

\begin{tabular}{|c|c|}
\hline Level & Factor \\
\cline { 2 - 2 } & $n$ \\
\hline 1 & 2.91776 \\
\hline 2 & 3.008 \\
\hline 3 & 3.09824 \\
\hline
\end{tabular}

After the random trial of 700 times, the probability density distributions of response surface 
model are obtained. They respective are: Weigh $\sim N\left(45.87,1.337^{2}\right)$, Resonate $\sim N(0.00105$, $0.0000299^{2}$ ). The maximum variation of spring mass is $2.91 \%$ and that of the spring vibration frequency is $2.85 \%$. The results meet the design requirement.

As will be seen: the robust performance of spring is poor (the maximum variation of the spring vibration frequency is beyond the allowable value) with random error. The robustness is improved based on the Taguchi method, after using the Monte Carlo method to determine the key factors (the steel wire diameter, the spring diameter) obtained by the Monte Carlo method.

\section{Conclusions}

The conventional deterministic optimization method has the limitations, and a tiny variation may lead to the design failure. In the engineering design only the deterministic optimization is not enough to ensure the robustness. The Monte Carlo method is used to test the results (solve based on the deterministic method) and decides whether or not to carry out robust design. The Taguchi method has improved the robustness of valve spring.

\section{Acknowledgements}

The research was funded by Chinese National Natural Science Foundation (51005167) and Tianjin University of Technology and Education Foundation (KJY11-07).

\section{Reference}

[1]Marseguerra M, Zio E, Podofillini I. Condition based Maintenance Optimization by Means of Genetic Algorithms and Monte Carlo Simulation. Reliability Engineering \& System Safety, 2002, 77(2):151 165

[2]ZHANG Lei, ZHANG Wenming, SHEN Yanhua. Robust design for locus generating steering mechanism based on Monte Carlo method. Journal of University of Science and Technology Beijing, 2006, 28(12):1174-1177

[3]ZHAO Juchu. Discussion once again on the improvement method of cylindrical spiral spring design. Machine design, 2003, 20(4):18-22

[4]Chen Lizhou. Robust design. Beijing: Mechanical industry press, 2000

[5]Disciuva M, Lomario D. A comparison between Monte Carlo and FORM in calculating the refiability of a composite structure. Composite Structures, 2003, 59(1):155-162 